\title{
RELATIONS BETWEEN TEXTURE AND ANISOTROPIC PROPERTIES : SOME APPLICATIONS TO LOW SYMMETRY MATERIALS
}

M. HUMBERT, F. WAGNER, M.J. PHILIPPE, C. ESLING

Laboratoire de Métallurgie des Matériaux Polycristallins, ISGMP - UFR MIM-Sciences

F-57045 Metz Cedex 01

\section{Introduction}

The experiment shows that most polycrystalline materials exhibit an anisotropy of physical properties, the origins of which can be found in the intrinsic properties of crystallites considered as single crystals but also in the structural parameters which characterize the polycrystalline state. For a specific use of a polycrystalline material, a given physical anisotropy must therefore be reached and controlled through well suited thermomechanical treatments which can strongly modify the polycrystalline state.In order to improve such processes or to understand the anisotropic behavior, models are usually developed in which the real behaviour of the polycrystal is considered as the result of combinations of the individual behaviour of the crystallites according to specific rules.

The efficiency of a model is appreciated inasmuch as it can reproduce the real behaviour of the material with the smallest uncertainty from an adequately reduced knowledge about structural parameters of the material. Among all these parameters the crystalline texture is mainly involved in the polycrystalline anisotropy (this is particularly obvious when measuring physical properties of sharp textured materials). Therefore models solely based on texture information have been developed and justified by the quality of their predictions. As a matter of fact successful predictions do not require the full texture information : it depends on the investigated property and on its modelling. This feature is of importance and allows reduction of the practical efforts for collecting data in order to obtain the minimal texture information required for a given model. Advantage can be taken of this opportunity especially when dealing with models applied to low symmetry materials to 
which this contribution is devoted.

II. Evaluation of polycrystalline physical properties

Theoretically the evaluation of polycrystalline properties requires the knowledge of the single crystal property and the full description of the aggregate by means of distribution and correlation functions $/ 1 \%$ In order to reduce the complexity of such calculations simplifying assumptions are often made.

Mean values

When the "interactions" between grains are not taken into account the simplest approximation of the effective properties $\mathrm{E}^{\mathrm{eff}}$ of a polycrystal consists in averaging the single crystal property e. This averaging is performed within the whole volume of the investigated sample :

$$
E^{\text {eff }} \approx\langle e\rangle=\frac{1}{V} \int_{V} e(r) d r^{3}
$$

In this expression e symbolically represents a tensor of appropriate type and rank describing a given anisotropic single crystal property.

Expression (1) can be evaluated from the O.D.F. in two integration steps :

$$
\langle e\rangle=\int d g \int_{v(g)} e(r) d r^{3}=\oint e(g) f(g) d g
$$

In other circumstances such as determining the $X$ Ray elastic constants this averaging is performed on the sole crystalline volumes in Bragg's condition.

$$
\bar{e}=\frac{\int_{g \in G} e(g) f(g) d g}{\int_{g \in G} f(g) d g}
$$

In this relation $G$ represents the orientations of crystallites in Bragg's position. 


\section{Self Consistent Approach}

The real behaviour of a polycrystalline aggregate obviously depends on the interaction between grains. One statistical way of taking the grain interaction into account is to solve the problem of the elastic inclusion embedded in the matrix considered by a homogeneous equivalent medium (HEM) of the same elastic properties. In this section we restrict representation to the elasticity. In elasticity the formalization and solution of this problem are due first to Eshelby /2,3/ and then to Kröner $/ 4 /$ and Kneer /5/. Applications to textured polycrystalline materials have been performed by Morris /6/ who outlines the important features of the method and describes the different steps of the calculation. From the equilibrium equation, the compatibility relation and the boundary conditions it is possible to express the relation between the local strain $\varepsilon$ in the inclusion and the macroscopic stress $\langle\sigma\rangle$

$$
\varepsilon=(I+u) S^{\text {eff }}\langle\sigma\rangle
$$

$S^{\text {eff }}$ is the effective compliance of the H.E.M., I the identity tensor of rank 4. The quantity $u$, related to the local $c$ and the effective $C^{\text {eff }}$ compliances, depends also on a tensor $E$ which is calculated from the equilibrium relation and in which the grain shape is taken into account :

$$
u=\left[c-C^{e f f}+E^{-1}\right]^{-1} E^{-1}-I
$$

The volume average of $u$ must theoretically be zero

$$
\langle u\rangle=\oint u(g) f(g) d g=0
$$

The effective stiffness $C^{\text {eff }}$ is calculated in an iterative procedure which reduces the value of $\left\langle u^{(n)}\right\rangle$ at each step $n$ according to the scheme:

$$
\left\langle u^{(n)}\right\rangle=\left\langle\left[c-C^{(n-1)}+\left(E^{(n-1)}\right)^{-1}\right] E^{(n-1)^{-1}}-I\right\rangle
$$


with $C^{(n)}=C^{(n-1)}+\gamma<u(n-1)>$

$\gamma$ is a scalar coefficient chosen for accelerating the convergency. It is assumed that $C^{(n)}$ is close to $C^{\text {eff }}$ when $n$ is great enough. At each step, the mean value $\left\langle u^{(n)}\right\rangle$ is calculated according to expression (6)

Expressions (2), (3), (6) and (7) are integrals which contain the O.D.F. $f(g)$. As a matter of fact the full information contained in $f(g)$ is not always entirely necessary. It depends on the nature of the investigated property.

\section{Properties represented by tensors of rank 4}

The elastic properties are represented by tensors of rank 4 . One way of determining the elastic behavior of polycrystals consists in calculating the mean values under Voigt's, Reuss's or Hill's assumptions as in expression (2).

\section{III.1. Mean values}

The components $E_{i j k l}$ of such a tensor expressed in a reference frame $K_{A}$ chosen by the experimenter are related to the components $E_{\text {opqr }}$ within the crystal reference frame by the relation :

$$
E_{i j k l}(g)=a_{i o}(g) a_{j p}(g) a_{k q}(g) a_{1 r}(g) E_{o p q r}
$$

where $g$ represents the orientation of the reference frame $K_{B}$ with respect to the reference frame $K_{A}$ and the $a_{m n}$ quantities are the direction cosines of the axis $n$ of $K_{B}$ with respect to the axis $m$ in $K_{A}$.

The product of direction cosines of relation (8) can be expressed by a series expansion of generalized spherical harmonics. If the tensor is an elastic tensor (compliance $S$ or stiffness C) one has additionnal symmetry relations.

$$
\mathrm{E}_{\mathrm{ijkl}}=\mathrm{E}_{\mathrm{ijlk}}=\mathrm{E}_{\mathrm{jilk}}=\mathrm{E}_{\mathrm{klij}}
$$

and the series expansion of $E_{i j k l}(g)$ involves only even generalized spherical harmonics (Jones $/ 7 /$, Backus /8/) up to a 
rank $\mathrm{L}_{\max }=4$ (Bunge $/ 9 /$ ) :

$E_{i j k l}(g)=\left[\sum_{1=0}^{4} \sum_{(2)}^{+1} \sum_{m=-1}^{+1} A_{1}^{n m^{*}}(i, j, k, l, o, p, q, r) T_{1}^{m n}(g)\right] . E_{o p q r}$

In this relation the $A_{1}^{n m}$ quantities are the series expansion coefficients. Because of the orthogonality of the generalized spherical harmonics and according to expression (2) the following integrals are equivalent:

$\left\langle E_{i j k l}\right\rangle=\int E_{i j k l}(g) f(g) d g=\int E_{i j k l}(g) \tilde{f}(g) d g=\int E_{i j k l}(g) \tilde{f}_{4}(g) d g$

$\tilde{f}$ is the even part of the O.D.F. $(f=\tilde{f}+\tilde{f})$ (Matthies /10/).

$\tilde{f}_{4}$ is the even part or the ODF represented by a series expansion truncated at $1_{\max }=4$.

$$
\tilde{f}_{4}(g)=\sum_{1=0(2)}^{4} \sum_{m} \sum_{n} C_{1}^{m n} T_{1}^{m n}(g)
$$

$\tilde{f}_{4}$ is the minimal texture information required for the mean value calculation of $E_{i j k l}$. This minimal texture information can be obtained by a minimal set of pole figures the number of which depends on the crystal symmetry as detailed in table $I$.

\begin{tabular}{|l|c|}
\hline $\begin{array}{l}\text { CRYSTAL } \\
\text { SYMMETRY }\end{array}$ & $\begin{array}{l}\text { POLE FIGURES } \\
\text { NUMBER }\end{array}$ \\
\hline CUBIC & 1 \\
\hline HEXAGONAL & 1 \\
\hline TRIGONAL & 2 \\
\hline TETRAGONAL & 2 \\
\hline ORTHORHOMBIC & 3 \\
\hline
\end{tabular}

Table I : Minimal number of pole figures required to acces $\mathrm{f}_{4}$ 
The last integral in expression (11) calculated by developing $\tilde{f}_{4}(g)$ and the product of direction cosines into series expansions gives as a result (Bunge $19 /$, Morris $/ 11 /$, Wenk 112/ :

$$
<E_{i j k} P=\left[\sum_{l=0}^{4} \sum_{m=-1}^{+1} \sum_{n=-1}^{+1} \frac{A_{l}^{m n^{*}}(i, j, k, 1,0, p, q, r) C_{l}^{m n}}{21+1}\right] E_{o p q r}
$$

In this case the $A_{1}^{m}$ * quantities can be calculated and stored into a data library. The calculation of the mean values $\left\langle\mathrm{E}_{\mathrm{ijkl}}\right\rangle$ can also be obtained by a direct numerical integration of expression (11). For that purpose it is necessary to sample Euler's space in accordance to the highest rank of the series expansion of the product Eijkl (g) $f(g)$. By using $\tilde{f}_{4}$ in relation (11), the angular sampling of Euler's space must not be higher than 22.5 degrees/13/.

\section{Case of the hexagonal symmetry}

The components of an elastic tensor of hexagonal symmetry are unchanged by a rotation of the reference frame around the $c$ axis of the crystal. Consequently the calculations of the mean elastic compliance or stiffness can be strongly simplified. As a matter of fact it has been shown /14/ that expression (11) is equivalent to

$\left\langle E_{i j k l}\right\rangle=\frac{1}{4 \pi} \int_{\varphi 1=0}^{2 \pi} \int_{\Phi=0}^{\pi} E_{i j k l}\left(\varphi_{1}, \Phi, 0\right) P_{00.1} \cdot\left(\Phi, \frac{\pi}{2}-\varphi_{1}\right) \sin \Phi d \Phi d \varphi_{1}(14)$

where $P_{00.1}$ is nothing else but the 00.1 pole density function.

\section{Example 1 : Young's modulus anisotropy}

For example this latter way of calculating has been applied to the prevision of the elastic behavior of a hexagonal $\mathrm{Zn}$ rolled sheet $/ 14 /$. The elastic compliance $<S_{i j k l} \gtrless_{R}$ according to Reuss's assumption and the elastic stiffness $\left\langle C_{i j k l}\right\rangle$ according to 
Voigt's assumption have been used to obtain Hill's mean value :

$$
\left\langle S_{i j k l}\right\rangle_{H}=\frac{1}{2}\left(\left\langle C_{i j k l}\right\rangle_{V}^{-1}+\left\langle S_{i j k l}\right\rangle_{R}\right)
$$

from which Young's moduli have been determined.

Figure 1 shows the good agreement between the predicted and the experimental data, which validates the use of the mean value procedure.

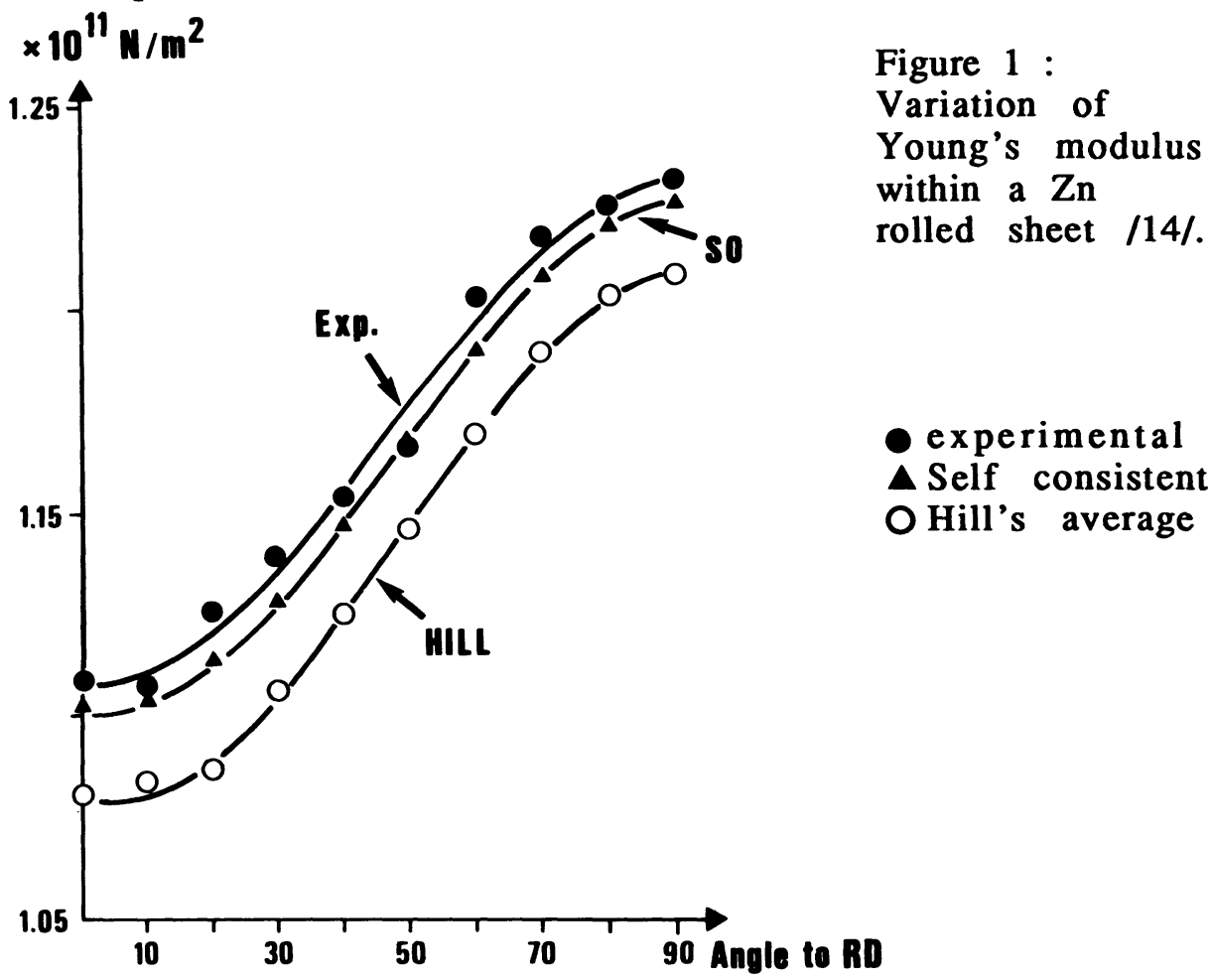

Example 2 : Anisotropy of $P$ and $S$ wave velocities

The calculation of elastic mean values is often used in the prediction of the anisotropy of the seismic $P$ and $S$ wave velocities. As a matter of fact Christoffel's equation

$$
\operatorname{det}\left|C_{i j k 1} m_{j} m_{k} P_{1}-\rho v^{2} P_{i}\right|=0
$$

relates the wave velocity $v$ propagating along the direction $\vec{m}$ 
with a polarization $\vec{P}$ with the elastic stiffness $C$ of the investigated single crystal, the density of which is $p$.

For example the expression $\rho v^{2}=C_{1111}$

relates the velocity of a $P$ wave propagating into the 100 direction of a single crystal.

For the polycrystal the mean value of (17) can be calculated according to Voigt's assumption

$$
\left.\rho<v^{2}\right\rangle=\langle\text { CVoigt }\rangle_{111}
$$

or Reuss's assumption

$$
\rho<v^{2}>=\left\langle\diamond_{\text {REUSS }}^{-1}\right\rangle_{111}
$$

and also Hill's assumption.

According to this scheme the wave velocity can be calculated whatever the direction in a polycrystalline aggregate. This way of calculating has been achieved by Seront and Mainprice /15/ for the prediction of the anisotropy of the $P$ wave velocity in a polycrystal of anorthosite considered here as orthorhombic in this calculation (plagioclase $90 \%+$ Olivine (orthorhombic) $10 \%)$.

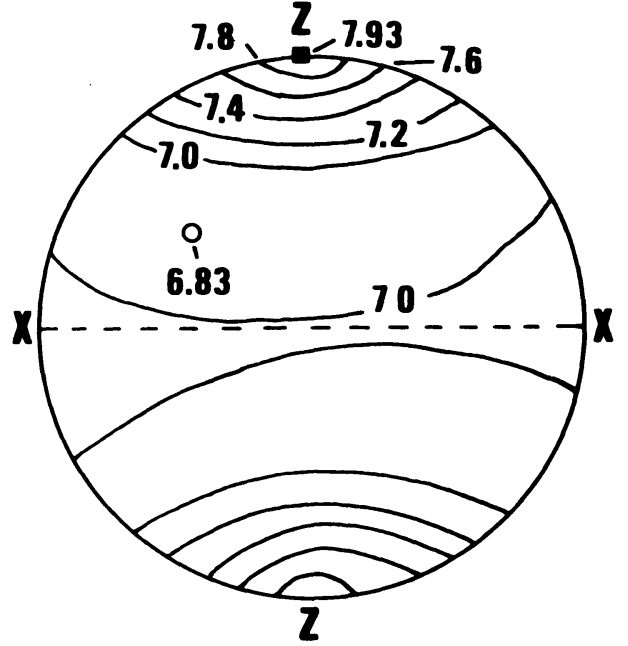

a)

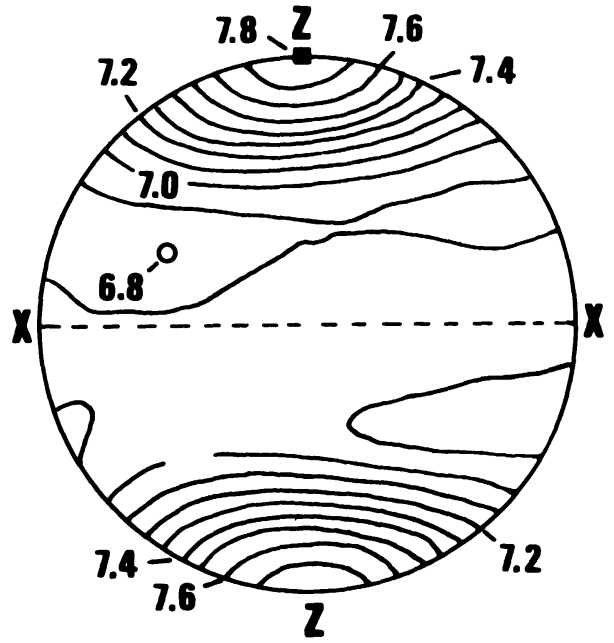

b)

Figure 2 : Comparison between the experimental (a) and the calculated (b) $\mathrm{P}$ wave velocities in anorthosite after /15/. 
Figure 2 shows the experimental data (a) as well as the predicted data (b) when Voigt's averaging is used. In that case the experimental and predicted velocities agree within 0.1 $\mathrm{Km} / \mathrm{s}$.

\section{III.2 Self-consistent approach}

In that scheme the following integral must be calculated at each step :

$$
<u^{(n)}>=\oint u^{(n)}(g) f(g) d g
$$

If $u^{(n)}(g)$ can be considered as an even quantity $/ 13 / \mathrm{f}(\mathrm{g})$ can be replaced by the even part $\tilde{f}(\mathrm{~g})$. In case of hexagonal symmetry, the value of the elastic stiffness $c(g)$ as well as the value of the quantity $u^{(n)}$ do not depend on a rotation around the $\mathrm{c}$ axis. In that case expression (19) reads /13/ :

$\left\langle U_{i j k 1}^{(n)}\right\rangle=\frac{1}{4 \pi} \int_{\varphi 1=0}^{2 \pi} \iint_{\Phi=0}^{\pi} u_{i j k 1}^{(n)}\left(\varphi_{1}, \Phi, 0\right) P_{00.1} \cdot\left(\Phi, \frac{\pi}{2}-\varphi_{1}\right) \sin \Phi d \Phi d \varphi_{1}$

This expression shows that the minimal texture information required for this calculation corresponds to the pole figure of the $c$ axis. This opportunity provided by the hexagonal lattice symmetry considerably reduces the computation time. Figure 1 shows the anisotropy of Young's moduli, predicted for a $\mathrm{Zn}$ rolled sheet, which has been calculated according to the previous self-consistent scheme. The predicted data and the experimental data are very close to each other $/ 14 /$ as well as to the results obtained under Hill's assumption.

\section{III.3. Averaging on restricted orientations}

Another important application in which the determination of elastic constants is necessary is the stress analysis of textured materials by $X$ ray diffraction. Expression (3) describes an averaging on a path of given orientations. This method is based on the shift of the diffraction lines due to the modification of the lattice parameters induced by the stress which is to be determined $/ 16,17 /$. In figure 3 the direction $\vec{y}$ corresponds to the normal of diffracting lattice planes of a grain in orientation $g$. The variation of the Bragg angle $\Delta \theta_{\mathrm{hkl}}$ can be 
linked to the relative variation of the interreticular distances of lattice planes, the normals of which are parallel to the direction $\vec{y}$ in the reference frame $K_{A}$. This relative variation can be expressed by $\varepsilon_{\mathrm{zz}}^{\prime}$ in the intermediate reference frame K'.

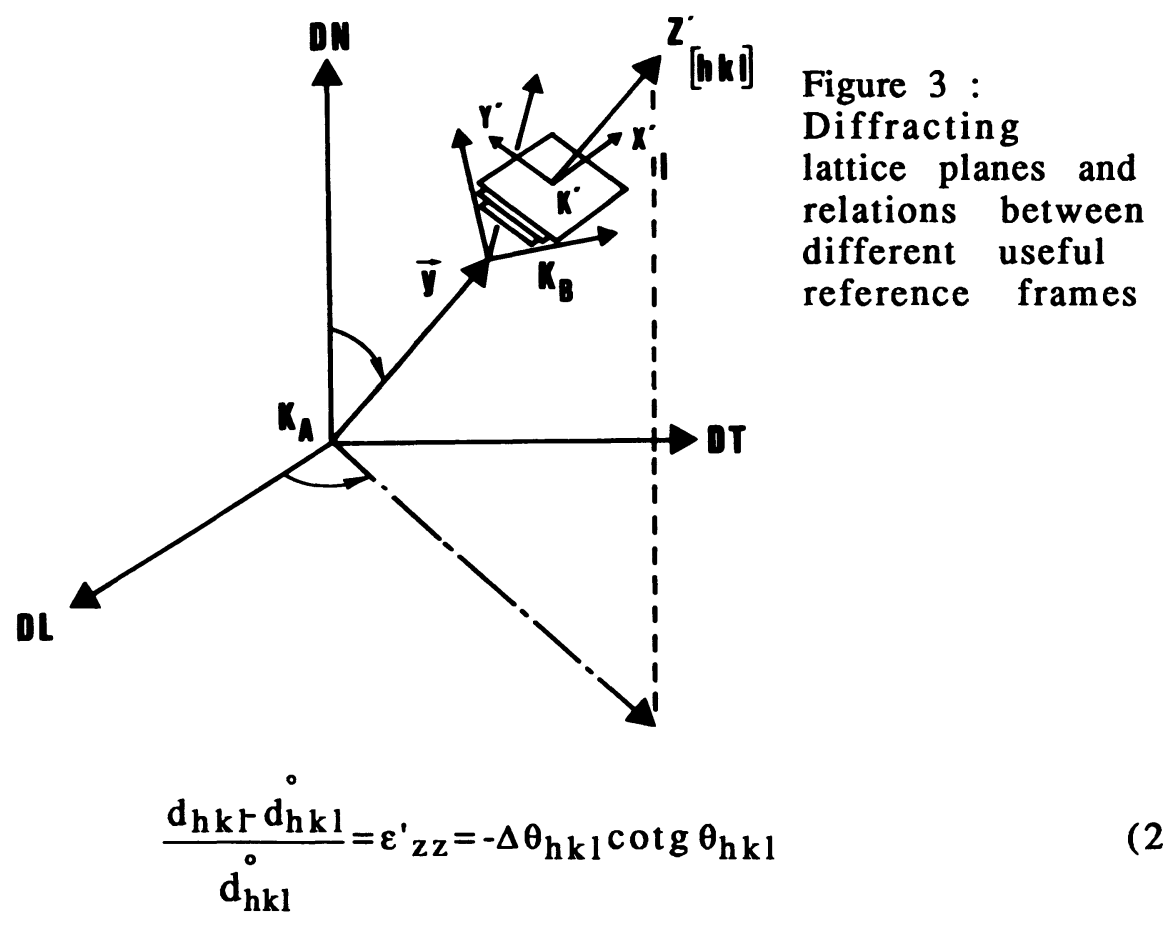

The strain $\varepsilon^{\prime}{ }_{z z}$ is related (by the elastic compliance of this grain) to the elastic stress $\sigma^{\prime}$ undergone by this grain $\left(\varepsilon^{\prime}, \sigma^{\prime}, S^{\prime}\right.$ being expressed in the $K^{\prime}$ reference frame).

$$
\varepsilon_{\mathrm{zz}}^{\prime}=S_{\mathrm{zztp}}^{\prime} \sigma_{\text {tp }}^{\prime}
$$

The following scheme gives the relations between the orientations of the macroscopic reference frame $K_{A}$, the intermediate reference frame $K^{\prime}$ and the microscopic reference frame $K_{B}$

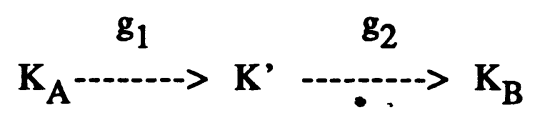


The elastic compliance $S^{\prime}$ can be given in the traditional microscopic reference frame $K_{B}$.

$$
S_{z z t p}=a_{z i}\left(g_{2}^{-1}\right) a_{z j}\left(g_{2}^{-1}\right) a_{t k}\left(g_{2}^{-1}\right) a_{p l}\left(g_{2}^{-1}\right) S_{i j k l}=T_{z z t p i j k l}\left(g_{2}^{-1}\right) S_{i j k l}
$$

In fact the mean shift of the diffraction line corresponds to the mean of the relative variations of interreticular lattice planes of grains in Bragg's position. Under Reuss's assumption this fact can be expressed by relation:

$$
\overline{\varepsilon_{z z}^{\prime}}=\overline{S_{z z t p}^{\prime}} \overline{\sigma_{t p}^{\prime}}
$$

from which stresses can be drawn.

The mean value of the compliance is performed on all the grains with the pole $\vec{h}$ parallel to $\vec{y}$; therefore $S_{\text {zztp }}$ reads

$$
\overline{S_{z z t p}}=\frac{\int_{0}^{2 \pi} T_{z z t p i j k l}\left(g_{2}^{-1}\right) f\left(g_{2} g_{1}\right) d \lambda_{2} S_{i j k l}}{\int_{0}^{2 \pi} f\left(g_{2} g_{1}\right) d \lambda_{2}}
$$

In reality, the mean value must be calculated also on the grains for which the pole $\overrightarrow{-h}$ is parallel to $\vec{y}$. Therefore it appears that the calculation of the mean value $\overline{S^{\prime}}$ requires the knowledge of the complete ODF (Brakman /18/). This remains true whatever the assumption (Reuss, Voigt, Hill, Kröner) and the symmetry of the investigated material.

\section{PROPERTIES REPRESENTED BY TENSORS OF SECOND BANK}

\section{IV.1. Mean values}

The polycrystal mean values are calculated in the same way as for the properties represented by tensor of fourth rank (see relation (11)) :

$$
\ll e_{i j}=\int e_{i j}(g) f(g) d g=\left[\int a_{i o}(g) a_{j p}(g) f(g) d g\right] e_{o p}
$$

where the $a_{m n}$ 's are direction cosines. 
Apart from the triclinic lattice symmetry $f(g)$ can be replaced by $\tilde{f}_{2}(g)$ which corresponds to the information contained in the even part of the O.D.F. up to rank $1_{\max }=2$ which can be obtained from a pole figure (apart for the triclinic symmetry). Without going into details the calculation of the mean values can be performed either by discretization of integral (26) or by replacing each function by its series expansion on spherical harmonics.

\section{Special cases}

For hexagonal, trigonal and tetragonal lattice symmetries the tensors of rank 2 present the following form :

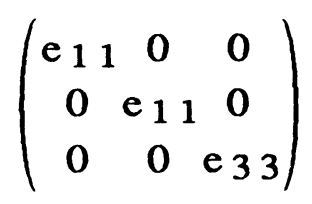

It is easily shown that $e_{i j}(g)$ does not depend on the third Euler angle $\varphi_{2}$ :

$$
e_{i j}(g)=e_{i j}\left(\varphi_{1}, \phi, \varphi_{2}\right)=e_{i j}\left(\varphi_{1}, \phi, 0\right)
$$

and therefore the mean value can be calculated in the following way.

$$
<e_{i j}>\int_{\varphi_{1}}^{2 \pi} \int_{\phi=0}^{\pi} e_{i}\left(\varphi_{1}, \phi, 0\right)\left[\int_{\varphi_{2}=0}^{2 \pi} f\left(\varphi_{1}, \phi, \varphi_{2}\right)\right] \sin \phi d \phi d \varphi_{1} \frac{1}{8 \pi^{2}}
$$

The partial integration in $\varphi_{2}$ of $\tilde{f}(g)$ is proportional to the density function $\operatorname{Poo.1}(\vec{y})$. Therefore for these special symmetries the mean values can be numerically calculated from the P00.1( $\vec{y})$ pole density function :

$<\mathrm{e}_{\mathrm{ij}}>\frac{1}{4 \pi} \int_{\varphi_{1}}^{2 \pi} \int_{\phi=0}^{\pi} \mathrm{e}_{\mathrm{ij}}\left(\varphi_{1}, \phi, 0\right) \mathrm{P}_{00.1}\left(\phi, \varphi_{1}-\pi / 2\right) \sin \phi \mathrm{d} \phi \mathrm{d} \varphi_{1}$

\section{Anisotropy parameters}

When the polycrystalline aggregate has moreover an 
orthohombic symmetry the corresponding mean value is reduced to :

$$
<e_{i p} p=\left(\begin{array}{cccc}
\left\langle e_{1} l\right. & & & \\
& <e_{22}> & \\
& & <e_{33}>
\end{array}\right)
$$

the mean diagonal terms being expressed by :

$$
\left\langle e_{i} p=\int a_{i d}^{2}(g) \tilde{f}_{2}(g) d g e_{o o}\right.
$$

By taking the properties of the direction cosines $\left(a_{i 1}^{2}+a_{i 2}^{2}+a_{i 3}^{2}=1\right)$ into account expression (32) reads :

$$
<e_{i} p=e_{11}+\left(e_{3}-e_{1}\right) \int \stackrel{2}{a_{i}}(g) \tilde{f}(g) d g
$$

The integrals of expression (33) are known as anisotropy parameters $\mathrm{Fi} / 19 /$ which can be determined from the 00.1 pole density fonctions :

$F_{1}=\frac{1}{4 \pi} \int_{\beta=0}^{2 \pi} \int_{\alpha=0}^{\pi} P_{00.1}(\alpha, \beta) \cos ^{2} \beta \sin ^{3} \alpha d \alpha d \beta$

$F_{2}=\frac{1}{4 \pi} \int_{\beta=0}^{2 \pi} \int_{\alpha=0}^{\pi} P_{00.1}(\alpha, \beta) \sin ^{2} \beta \sin ^{3} \alpha d \alpha d \beta$

$F_{3}=\frac{1}{4 \pi} \int_{\beta=0}^{2 \pi} \int_{\alpha=0}^{\pi} P_{00.1}(\alpha, \beta) \cos ^{2} \alpha \sin \alpha d \alpha d \beta$

or related to the $\mathrm{C}_{2}$ coefficients of the ODF $\mathrm{f}(\mathrm{g}) / 20$ /

$$
\begin{aligned}
& F_{1}=\frac{1}{3}-\frac{1}{15} C_{2}^{10}+\frac{1}{5} \sqrt{\frac{2}{3}} \operatorname{Re}\left[C_{2}^{12}\right] \\
& F_{2}=\frac{1}{3}-\frac{1}{15} C_{2}^{10}-\frac{1}{5} \sqrt{\frac{2}{3}} \operatorname{Re}\left[C_{2}^{12}\right] \\
& F_{3}=\frac{1}{3}+\frac{2}{15} C_{2}^{10}
\end{aligned}
$$


The different relations of the present section can be used for evaluating a lot of polycrystalline properties, some examples of which are proposed in the following part.

Example 3 : Thermat expansion of Uranium

The thermal expansion coefficients of an uranium single crystal which is orthorhombic are anisotropic. Different determinations have been performed. Stürken and Croach /21/ have predicted the thermal expansion of an uranium rolled sheet from the following single crystal data determined at room temperature :

$$
\begin{aligned}
& \alpha_{11}=25,3 \times 10^{-6} / \mathrm{C}^{\circ} \\
& \alpha_{22}=-0,2 \times 10^{-6} / \mathrm{C}^{\circ} \\
& \alpha_{33}=22 \times 10^{-6} / \mathrm{C}^{\circ}
\end{aligned}
$$

Table II shows the good agreement between the experimental and the calculated polycrystalline thermal expansion parameters.

\begin{tabular}{|l|l|l|}
\hline & $\begin{array}{l}\text { Experimental } \\
\text { values }\end{array}$ & $\begin{array}{l}\text { Calculated } \\
\text { values }\end{array}$ \\
\hline$\alpha_{11}^{p}$ & $9,2 \times 10^{-6} / \mathrm{C}^{\circ}$ & $9,07 \times 10^{-6} / \mathrm{C}^{\circ}$ \\
\hline$\alpha_{22}^{\mathrm{p}}$ & $19,9 \times 10^{-6} / \mathrm{C}^{\circ}$ & $19,12 \times 10^{-6} / \mathrm{C}^{\circ}$ \\
\hline$\alpha_{33}^{\mathrm{p}}$ & $21 \times 10^{-6} / \mathrm{C}^{\circ}$ & $20,6 \times 10^{-6} / \mathrm{C}^{\circ}$ \\
\hline
\end{tabular}

Table II

\section{Example 4: Dielectric susceptibility}

The dielectric susceptibility which relates the electric field to the electric polarization is generally anisotropic for low 
symmetry materials.

By using the dielectric susceptibility of calcite single crystal (trigonal).

$\left(\chi_{11}=\chi_{22}=7.5, \chi_{33}=7.0\right)$. Dreyer $/ 22 /$ has calculated from 327 orientations the dielectric susceptibility of a marble sample which has been plastically deformed $/ 23 /$.

The calculated susceptibility along the normal of the sample $\left\langle\mathrm{X}_{33}\right\rangle=7,12$ was very close to the experimental value.

\section{Example 5 : Irradiation growth of zircaloy}

When polycrystalline zircaloy products are submitted to a high flux of neutrons, one observes variations in their dimensional parameters. This effect is called irradiation growth. According to BUCKLEY's first investigations /24/ the growth induced by neutron irradiation on hexagonal materials is highly anisotropic and can be seen as a contraction along the $C$ axis and an isotropic expansion within the basal plane, as shown on figure 4 :

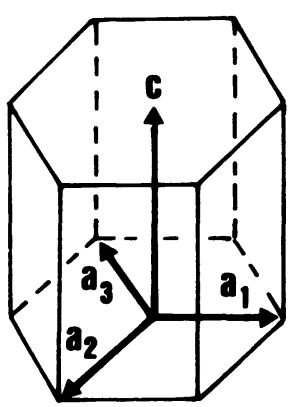

a)

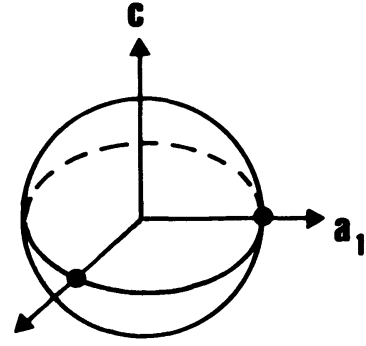

b)

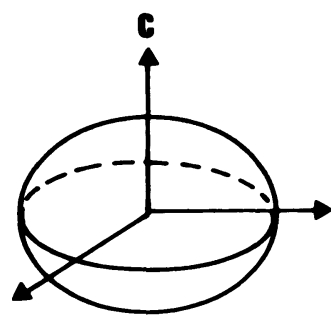

6)

Figure 4 : Model of deformation induced by irradiation

a) single crystal

b) single crystal sphere before irradiation

c) crystalline volume after irradiation

Therefore the irradiation growth of a single crystal can be conveniently represented at a given fluence by a strain tensor $\left(\varepsilon_{\mathbf{i j}}\right)$.

One further assumes that the polycrystalline behaviour can be evaluated by averaging the contribution of all the crystallites of the polycrystal. Therefore the polycrystalline growth can be expressed by : 


$$
\left.<\varepsilon_{i j}\right\rangle=\int \varepsilon_{i j}(g) \tilde{f}_{2}(g) d g
$$

The strain $\varepsilon(\vec{y})$ in a given direction $\vec{y}$ within the sample is given by the relation (20).

$$
\varepsilon(\vec{y})=\varepsilon_{11}+\left(\varepsilon_{33}-\varepsilon_{11}\right) w(\alpha, \beta)
$$

where $w(\alpha, \beta)$ is a function depending on the texture. This previous relation can be used in several ways. It allows to calculate the macroscopical deformation of the polycrystal provided that the single crystal characteristics and little but sufficient information on the texture be known. Conversely single crystal growth characteristics can be deduced from the texture information and from the macroscopical deformations. These growth-characteristics can be re-used in relation (37), this time for evaluating the behaviour of materials, the metallurgical state of which is close to that of the reference material. Very often, the crystallographic texture is not involved, alone, in the irradiation growth. Therefore one has to take other metallurgical parameters into account. (CARPENTER /25/, ADAMS $/ 26 /$ ).

\section{IV.2 Effective values}

A lot of works have been devoted to the calculation of physical properties such as dielectric or thermal expansion properties, but very few for textured materials.

Example 6: Thermal expansion anisotropy of polycrystals.

In thermal expansion the deformation of grains is limited by the surrounding elastic matrix which prohibits the incompatible deformations, causing internal stresses. The mean value of these internal stresses which can be related to the differences of thermal expansion between the polycrystal and the single crystal must be null.

This condition allows Kneer in his thesis /5/ (page 108 ) to express the deformation of the polycrystal from the single crystal deformation induced by temperature rise. The relation adapted for textured materials reads :

$$
\left.\mathrm{A}=\left\langle\mathrm{C}(\mathrm{I}-\mathrm{w}) \mathrm{m}^{*}\right\rangle^{-1}<\mathrm{C}(\mathrm{I}-\mathrm{w}) \mathrm{m} * \alpha\right\rangle
$$


A $(\operatorname{resp} \alpha)$ is the tensor of thermal expansion of the polycrystal (resp. single crystal).C is the effective stiffness of the polycrystal. $w$ is a tensor which solves the elastic inclusion problem ; it is related to the quantity $E$ in expression (5) and takes the grain shape into account.

$\mathrm{m}^{*}$ is defined by :

$$
\mathrm{m}^{*}=[\mathrm{I}-\mathrm{sC}(\mathrm{I}-\mathrm{w})]
$$

where $\mathbf{s}$ is the local compliance.

The different averagings of expression (38) are calculated in the same way as in expression (2).

This method of calculation has been used for predicting the thermal expansion anisotropy of a $\mathrm{Zn}$ rolled sheet $/ 27 /$.

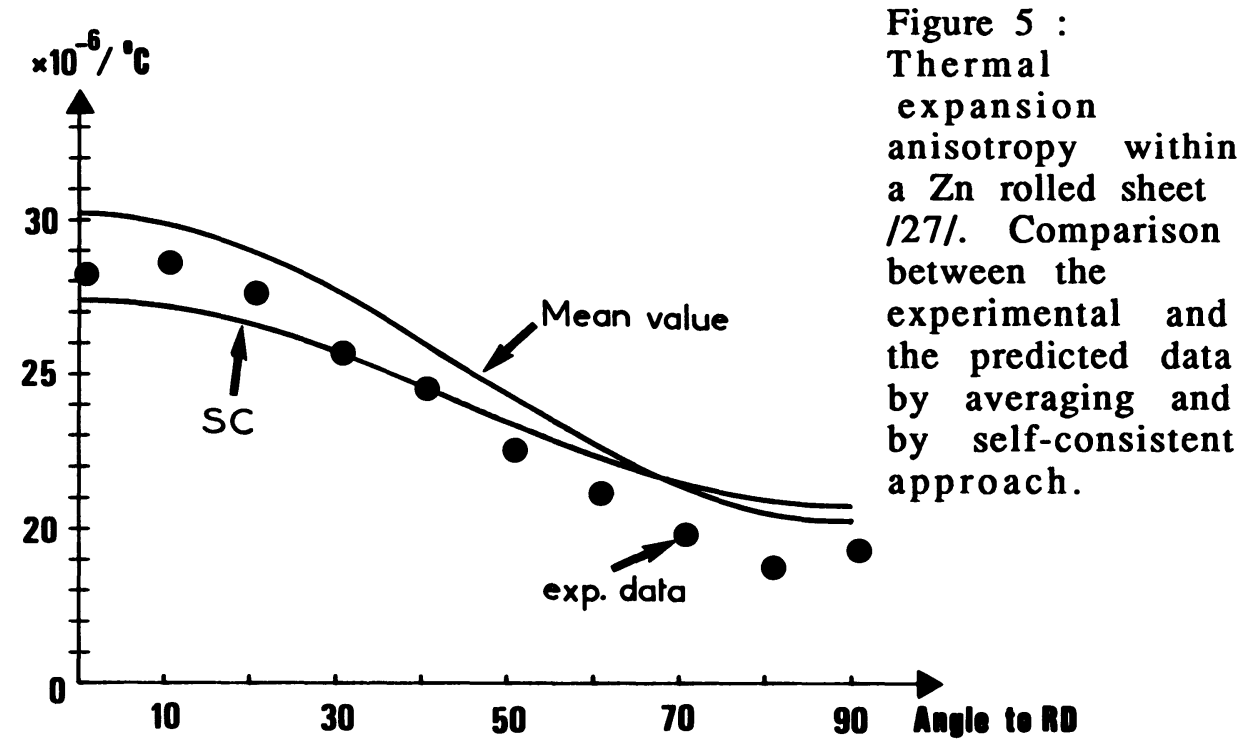

Figure 5 shows the experimental thermal expansion coefficients measured in different directions from the rolling direction as well as the predicted mean values and the predicted effective values. All the results are contained within a very narrow band (the extreme values are distant of about 10\%). The effective values calculated for elongated grains are always smaller than the mean values because the part of grain strains 
which is incompatible with the geometrical deformation of the matrix induces internal stresses and not strains. The evaluation of effective values requires complex calculations for results which are very close to the mean values easily obtained by using the general relation (26) or relation (30) for hexagonal symmetry materials.

\section{CONCLUSION}

The prevision of polycrystalline properties requires a quantitative statistical information about the polycrystal. Most of the time this information is limited to the texture. The modellings based on the volume averaging of single crystal property only requires a restricted part of texture information which can be described by few even terms of the series expansion of the O.D.F. This reduced texture information is determined from few pole figures, the number of which depends on the lattice symmetry of the investigated material. For some special symmetries and properties (hexagonal symmetry and elasticity, hexagonal, trigonal, tetragonal symmetries and properties represented by a second rank tensor) the prevision can be directly performed from the 00.1 pole figure data. The volume averaging-type previsions are generally very close to the real behavior of the materials.

A more sophisticated way of evaluating the effective values consists in the self-consistent approach which models the behavior of the materials by taking the grain interactions into account. This method requires the volume averaging of different quantities which describe the relations between the local and the macroscopical behaviors. Theoretically the complete ODF should be used. Some tests on hexagonal material show that the even part of the ODF is sufficient. As a matter of fact the results given by this method are in most cases, very close to the prediction obtained by simple averaging.

The determination of $X$ ray elastic constants which is generally based on the averaging of restricted orientations demands the complete texture information.

\section{References}

/ $1 /$ BERAN M. "Statistical continuum Theories" Wiley (Interscience), New York.

/ 2 / ESHELBY J.D., Proc. R. Soc. A241, 376 (1957)

/ 3 / ESHELBY J.D., Proc. R. Soc. A252, 561 (1959)

/ 4 / KRONER E., J. Phys., 151, 504 (1958) 
/5/ KNEER, Dissertation, Bergakademie Clausthal, Technische Hochschule (1964)

/ 6 / MORRIS P.R., Int. J. Engng. Sci. 8, (1970) 49-61

/7/ JONES H.N. "Spherical harmonics and tensors for classical field theory" Research studies press (1985)

/ 8 / BACKUS G.E., Rev. Geophys Space Phys. 8, (1970) 633-671

19/ BUNGE H.J., Texture Analysis in Materials Sciences Butterworths, London (1982)

/ 10 / MATTHIES S., Phys. Statis. Solidi B93 (1979), K135

/ 1 1/ MORRIS P.R. and HECKLER A.J., Trans. TMS-AIME 245 (1968) p. $1877-1881$

/ 12 / JOHNSON G.C. and WENK H.R., J. appl. Phys. 1 (1986) 38683875

/13/ DIZ J. and al. to be published

/14/ HUMBERT M. and al. to be published

/ 15 / SERONT B., MAINPRICE D. and CHRISTENSEN to be published in J. Geophysical Research.

/ 1 6/ DOLlE H., J. Appl. Cryst. 12 (1979), 489-501

/ 17 / HAUK V., Adv. X-Ray Anal. 27 (1984), 101-120

/ 1 8 / BRAKMAN C.M., J. Appl. Cryst. 18 (1985), 279-295

/19/ KEARNS J.J., WAPD-TM-472, TID-4500, Bettis Atomic Power Lab. Pittsburgh P.A. (Nov. 1965)

/ 20 / HUMBERT M., DIZ J., BARD. J., ESLING C., to be published 121 / STURKEN E.F. and CROACH J.W., Met. Trans. Met. Soc. AIME 227 (1963), 934-940

/2 2/ KARL F. and KERN H., Contr. Miner. Petr. 18 (1968), 199-224

/23/ DREYER N., Bergbauwissenschaften 14, (1970) 336-374

/ 24 / BUCKLEY S.N. J. Inst. Metals 97 (1969) 61

/ 25 / CARPENTIER G.J.C., ZEE R.H. and ROGERSON A., J. of Nucl. Mat. 159 (1988), 86-100

/ 26 / ADAMS B.L. and CLEVINGER, J. of Nucl. Mat. 90 (1980), 75-88

$127 /$ M. HUMBERT, J. WEGRIA and C. ESLING, This volume. 